\title{
Spatial Analysis of Phosphatidylinositol Molecular Species in Pork Chop Tissues Using Matrix-assisted Laser Desorption/ionization-Mass Spectrometry
}

\section{Imaging}

Hirofumi Enomoto ${ }^{1,2,3 *}$, Shiro Takeda ${ }^{4}$ and Hajime Hatta ${ }^{5}$

${ }^{1}$ Department of Biosciences, Faculty of Science and Engineering, Teikyo University, 1-1 Toyosatodai, Utsunomiya 320-8551, JAPAN

${ }^{2}$ Division of Integrated Science and Engineering, Graduate School of Science and Engineering, Teikyo University, 1-1 Toyosatodai Utsunomiya 320-8551, JAPAN

${ }^{3}$ Advanced Instrumental Analysis Center, Teikyo University, 1-1 Toyosatodai, Utsunomiya 32-8551, JAPAN

${ }^{4}$ Department of Animal Science and Biotechnology, School of Veterinary Medicine, Azabu University, 1-17-71 Fuchinobe, Chuo-ku, Sagamihara 252-5201, JAPAN

${ }^{5}$ Department of Food and Nutrition, Faculty of Home Economics, Kyoto Women's University, 35 Kitahiyoshi-cho, Imakumano, Higashiyama-ku, Kyoto 605-8501, JAPAN

\begin{abstract}
Matrix-assisted laser desorption/ionization-mass spectrometry imaging (MALDI-MSI) is a powerful technique for visualizing lipids in biological tissues. Phosphatidylinositol (PI), a phospholipid in pork, is a major source of inositol in animal-derived foods believed to be protective against diseases related to pregnancy and cancer. However, the distribution of PI molecular species in pork is not well understood. Here, we performed MALDI-MSI analysis to investigate the distribution and composition of PI molecular species in pork chop comprising Longissimus thoracis et lumborum muscle (loin), intermuscular fat tissue, transparent tissue, and spinalis muscle. Twelve diacyl-PI molecular species were identified using liquid chromatography-electrospray ionization-tandem mass spectrometry (MS/MS) and MALDI-MS/MS analysis and visualized using MALDI-MSI. Spinalis muscle had the highest amount of identified PI molecular species, followed by loin, transparent tissue, and intermuscular fat tissue. The diacyl-PI molecular species containing hexadecadienoic, oleic, linoleic and eicosadienoic acids at the $s n-2$ position were mainly abundant in the loin and spinalis muscle, whereas those containing mead, arachidonic, docosatetraenoic, and docosapentaenoic acids at the $\mathbf{s} n-\mathbf{2}$ position were mainly abundant in both muscles as well as transparent tissues. Notably, the balance of PI molecular species differed among the tissues depending on fatty acid compositions at the $s n-2$ position. These results suggested that MALDI-MSI is a promising tool for assessing the association between individual pork tissues and the protective effects of PI molecular species against diseases related to pregnancy and cancer. To the best of our knowledge, this is the first report showing tissue-specific distributions of PI molecular species in pork chop using MALDI-MSI.
\end{abstract}

Key words: phosphatidylinositol, pork, mass spectrometry imaging, matrix-assisted laser desorption/ionization, liquid chromatography-tandem mass spectrometry

1 Introduction

Liquid chromatography (LC) -electrospray ionization (ESI)-tandem mass spectrometry (MS/MS), commonly used to analyze various molecules ${ }^{1,2)}$, can analyze nearly all lipid classes with high sensitivity based on differences in fatty acid compositions. Therefore, this technique is useful for qualitative and quantitative analyses of molecular species of lipids. However, spatial analysis of lipids using this technique is complicated because it requires lipid extraction from samples. Matrix-assisted laser desorption/ ionization-mass spectrometry imaging (MALDI-MSI) is a powerful technique for visualizing molecules in biological tissues $^{3-9)}$. MALDI-MSI has been used to simultaneously investigate the content and spatial distribution of lipids at

\footnotetext{
*Correspondence to: Hirofumi Enomoto, Department of Biosciences, Faculty of Science and Engineering, Teikyo University, 1-1 Toyosatodai, Utsunomiya 320-8551, JAPAN

E-mail: enomoto@ nasu.bio.teikyo-u.ac.jp

Accepted March 29, 2021 (received for review January 28, 2021)

Journal of Oleo Science ISSN 1345-8957 print / ISSN 1347-3352 online

http://www.jstage.jst.go.jp/browse/jos/ http://mc.manusriptcentral.com/jjocs

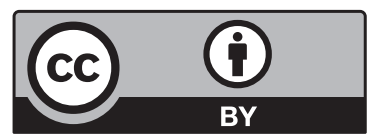




\section{H. Enomoto, S. Takeda and H. Hatta}

microscopic resolution without the need for antibodies, staining, or complicated preliminary procedures ${ }^{10-14)}$.

Meat is an important source of lipids, proteins, vitamins, and micronutrients ${ }^{15,16)}$. Pork is one of the most consumed meats worldwide. Phospholipids are major components of meat that affect its qualities including nutritional and health-related properties ${ }^{1,15,16)}$. Previously, we identified phosphatidylcholine and sphingomyelin molecular species as the major phospholipids in pork chop and visualized them using LC-ESI-MS/MS and MALDI-MSI ${ }^{12,13)}$. Phosphatidylinositol (PI), a major phospholipid in meat, is a component of the cell membrane bilayer and functions as a reservoir of polyunsaturated fatty acids (PUFAs), predominantly arachidonic acid (20:4). PUFAs have various health-promoting effects in individuals suffering from chronic diseas$\mathrm{es}^{17)}$. PIs are also a major source of animal food-derived inositol. Also known as a pseudo-vitamin (vitamin Bh or B8), inositol has been suggested to be protective against diseases related to pregnancy and cancer ${ }^{18-21)}$. However, the distribution of PI molecular species in meat is not well known.

In the present study, we aimed to identify PI molecular species in pork chop using LC-ESI-MS/MS and MALDI-MS/ MS analysis. Subsequently, we visualized and compared the amounts of PI molecular species in four different tissues and revealed characteristic distribution patterns of PI molecular species in the four different pork tissues.

\section{EXPERIMENTAL PROCEDURES}

\subsection{Materials}

Water, chloroform, methanol, acetonitrile, isopropanol, and ammonium formate were purchased from FUJIFILM Wako Pure Chemical Corp. (Tokyo, Japan), and 9-aminoacridine (9AA) was purchased from Sigma Aldrich (St Louis, MO, USA). Alpha-cyano-4-hydroxycinnamic acid (CHCA) was purchased from Tokyo Kasei Kogyo Co., Ltd. (Tokyo, Japan). A peptide calibration standard containing angiotensin II was purchased from Bruker Corp. (Billerica, MA, USA). All reagents and solvents used in the study were of analytical grade.

\subsection{Pork samples}

Pork chops (6-month-old female crossbred pigs, Duroc $\times$ Danish Landrace $\times$ Yorkshire) were purchased from a local supermarket one day after slaughter. The samples were stored in a $-80^{\circ} \mathrm{C}$ freezeruntil needed.

\subsection{Preparation of pork sections}

Pork sections were prepared as described in our previous study ${ }^{12,13)}$. Briefly, consecutive $10-\mu \mathrm{m}$ sections containing four different tissues; namely, Longissimus thoracis et lumborum muscle(loin), intermuscular fat tissue, trans- parent tissue, and spinalis muscle, were prepared using a cryostat(CRYOCUT CM1860; Leica Microsystems, Wetzlar, Germany). The frozen sections were mounted on indium tin oxide (ITO)-coated glass slides $\left(100 \Omega / \mathrm{m}^{2}\right.$; Matsunami Glass, Osaka, Japan), which were then placed in $50 \mathrm{~mL}$ centrifuge tubes containing silica gel and preserved at $-80^{\circ} \mathrm{C}$ until MALDI-MSI.

\subsection{Matrix vapor deposition}

Matrix vapor deposition/recrystallization was performed as previously described ${ }^{8)}$, with minor modifications. An ITO-coated glass slide loaded with a frozen section was taken out of the freezer and dried in a vacuum desiccator for 30 min. Matrix vapor deposition was performed using a vacuum deposition system (SVC-700TMSG, Sanyu Electron Co., Ltd., Tokyo, Japan). The glass slide was attached to the sample holder using adhesive tape, and 9AA powder (60 mg) was added on the matrix holder. The distance between the sample holder and the matrix holder was maintained at $8 \mathrm{~cm}$. After the vacuum pressure in the chamber reached $<5 \times 10^{-3} \mathrm{~Pa}, 9 \mathrm{AA}$ was heated from $240^{\circ} \mathrm{C}$ to $300^{\circ} \mathrm{C}$ for approximately $30 \mathrm{~min}$, until the entire powder had sublimed.

\subsection{MALDI-MSI and MS/MS analysis}

MALDI-MSI analysis was performed as described in our previous study ${ }^{7,8)}$. The 9AA-coated sections were applied to a MALDI-time of flight (TOF/TOF) instrument (UltrafleXtreme, Bruker) equipped with a 355-nm Nd:YAG laser at a repetition rate of $2000 \mathrm{~Hz}$. Data were collected in negative ion mode (reflector mode) at a step size of $100 \mu \mathrm{m}$. The laser diameter was set to medium size. The $m / z$ values ranging from 850 to 920 were measured. Calibration was performed externally before the measurements using the exact $m / z$ of both CHCA $[\mathrm{M}-\mathrm{H}]^{-}$ions $(m / z 188.03532)$ and angiotensin II $[\mathrm{M}-\mathrm{H}]^{-}$ions $(\mathrm{m} / z$ 1044.52725). All spectra were collected automatically. Normalization was performed based on the total ion current using FlexImaging 4.1 software (Bruker). Two-dimensional ion-density maps were also prepared using this software.

Three sections prepared from the same pork chop attached on the same ITO-coated glass slide were measured, and detection intensities in the loin, intermuscular fat tissue, transparent tissue, and spinalis muscle were obtained using the "region of interest" function of FlexImaging 4.1 software. The mean detection intensities of the PI molecular species identified in the four tissues were compared.

MALDI-MS/MS analyses were performed in collision-induced dissociation "LIFT" MS/MS mode, and the selected precursor and product ions were acquired. The MS/MS window was set to the precursor ion $m / z \pm 6$ Da. The MS/ MS spectra were analyzed using FlexAnalysis 3.4 software (Bruker) 


\subsection{Preparation of total lipid extracts}

Consecutive $10-\mu \mathrm{m}$ pork chop sections were collected (total $100 \mathrm{mg}$ ) in $1.5 \mathrm{~mL}$ micro tubes. Loin $(161 \mathrm{mg})$ and spinalis muscle (133 mg) were separated from the pork chop with a knife, and put in $2.0 \mathrm{~mL}$ micro tubes. Total lipid extract was prepared according to the Bligh and Dyer method $^{22)}$. After drying in a vacuum centrifugal evaporator (CVE-2200, Eyela Tokyo Rikakikai Co. Ltd., Tokyo, Japan), lipids were dissolved in $1 \mathrm{~mL}$ methanol:isopropanol (9:1, v/ v) and subjected to LC-ESI-MS and MS/MS analysis.

\subsection{LC-ESI-MS, and MS/MS analysis}

One microliter of the total lipid extract was injected into a Waters Acquity UPLC connected to a Waters Synapt XS quadrupole-TOF mass spectrometer equipped with an ESI ion source (Waters Corp., Milford, MA). A Waters ACQUITY UPLC BEH C18 reversed-phase column $(2.1 \times 100 \mathrm{~mm}$; $1.7-\mu \mathrm{m}$ particle size) was used at $40^{\circ} \mathrm{C}$. The mobile phase comprised solvent A[acetonitrile:water $(9: 1, \mathrm{v} / \mathrm{v})]$ and solvent B (isopropanol), both of which contained $10 \mathrm{mM}$ ammonium formate. The LC flow rate was $0.4 \mathrm{~mL} / \mathrm{min}$. The LC gradient conditions were as follows: 15\%-48\% B from 0 to $20 \mathrm{~min}, 90 \%$ B from 20 to $25 \mathrm{~min}$, and $15 \%$ B from 25 to $28 \mathrm{~min}$. The MS instrument was set to negative ion mode. Each total lipid extract was measured three times, and base peak intensity chromatogram of each exact $\mathrm{m} / \mathrm{z}$ value \pm 10 ppm was obtained using MassLynx software (Waters). Relative intensities (\%) were calculated from their peak areas, and shown as mean value \pm standard deviation $(n=3)$. To compare the total PI concentrations between loin and spinalis muscle, the mean detection intensities of total diacyl-PI molecular species were shown as per $1 \mathrm{mg}$ weight.

MS/MS spectra were collected at the exact $m / z$ values of PI molecular species ions \pm approximately 1 Da as the precursor ions using the same instrument. The trap and transfer collision energies were set at 45 and $2 \mathrm{eV}$, respectively. Data analysis was performed using MassLynx software (Waters).

\subsection{Statistical analyses}

In Figs. $3 a$ and $3 b$, the MALDI-MSI data are expressed as mean values \pm standard error $(n=3)$. The data were compared using one-way ANOVA, and post-hoc analysis was conducted using the Tukey's test. Statistical analyses were performed using the four tissues for each PI molecular species. Mean values with different letters indicate significant differences $(p<0.05)$. In Fig. 3c, asterisks $(*)$ in the LC-ESI-MS data indicate significant differences $(p<0.05)$ determined by Student's $t$-test.

\section{Results and Discussion}

\subsection{Mass spectra of pork chop sections}

Portions of the pork chops (Fig. 1a) that contained four different tissues; namely, loin, intermuscular fat tissue, transparent tissue, and spinalis muscle (Fig. 1b), were selected for analysis. We used 9AA as a matrix because it is commonly used for the analysis of PI molecular species using negative ion mode of MALDI-MSI ${ }^{14)}$. Vapor deposition was used as the matrix coating method because it forms uniform and fine matrix crystals on the sections. Under experimental conditions, PI molecular species are generally detected as $[\mathrm{M}-\mathrm{H}]^{-}$ions and odd-numbered peaks in the $m / z$ rage from 800 to 950 in animal tissues ${ }^{14)}$. Several peaks with odd numbers were observed in the mass spectra of the four different tissues (Figs. 1c-1f), indicating the detection of PI molecular species in the pork chop sections. In addition, the mass spectra patterns of the different four tissues appeared different from one another, implying that the concentrations of PI molecular species among these tissues were dissimilar.

\subsection{Identification of PI molecular species in pork chop sections}

To identify the PI molecular species detected in pork chops by MALDI-MSI, total lipid extracts were analyzed using LC-ESI-MS/MS in negative ion mode. PI comprises a glycerol backbone with fatty acids at the $s n-1$ and $s n-2$ positions and an inositol phosphate head group at the $s n-3$ position (Fig. 1g). In general, the fatty acids at the $s n-1$ position are palmitic (16:0), stearic (18:0), and oleic (18:1) acids, whereas those at the $s n-2$ position are PUFAs ${ }^{23)}$. In animal tissues, PI molecular species are dominantly present in a diacyl form. Inositol phosphate $[\mathrm{M}-\mathrm{H}]^{-}$ion (exact $m / z 241.013$ ) and two fatty acid $[\mathrm{M}-\mathrm{H}]^{-}$ions are commonly detected as intense fragment ion peaks in MS/ MS analysis of diacyl-PI molecular species (Fig. 1g) ${ }^{1,2)}$. For example, in the MS/MS spectrum of the precursor ion at $\mathrm{m} /$ $z$ 857.517, a fragment ion peak was detected at $\mathrm{m} / z$ 241.013, suggesting that the ion was diacyl-PI $[\mathrm{M}-\mathrm{H}]^{-}$. Notably, fragment ion peaks at $m / z 255.232$ and 303.233 corresponded to the 16:0 and arachidonic (20:4) acid[M$\mathrm{H}]^{-}$ions. Therefore, the peak at $m / z 857.517$ was identified as a PI(diacyl 16:0/20:4) [M- H $]^{-}$ion(Table 1). Similarly, in the MS/MS spectrum of the precursor ion at $\mathrm{m} / \mathrm{z}$ 861.553, fragment ion peaks were detected at $\mathrm{m} / \approx 241.013$, 279.233 , and 283.266 (Table 1). The fragment ion peaks at $\mathrm{m} / \approx 279.233$ and 283.266 corresponded to linoleic (18:2) and 18:0 $[\mathrm{M}-\mathrm{H}]^{-}$ions. Thus, the peak at $\mathrm{m} / z 861.553$ was assigned as a PI(diacyl 18:0/18:2) $[\mathrm{M}-\mathrm{H}]^{-}$ion (Table 1). In other PI molecular species, fragment ion peaks at $\mathrm{m} / \mathrm{z}$ 251.203, 281.249, 301.220, 303.233, 305.248, 307.263, 329.250 , and 331.266, corresponding to hexadecadienoic (16:2), 18:1, eicosapentaenoic (20:5), 20:4, mead (20:3), eicosadienoic (20:2), docosapentaenoic (22:5), and docosa- 

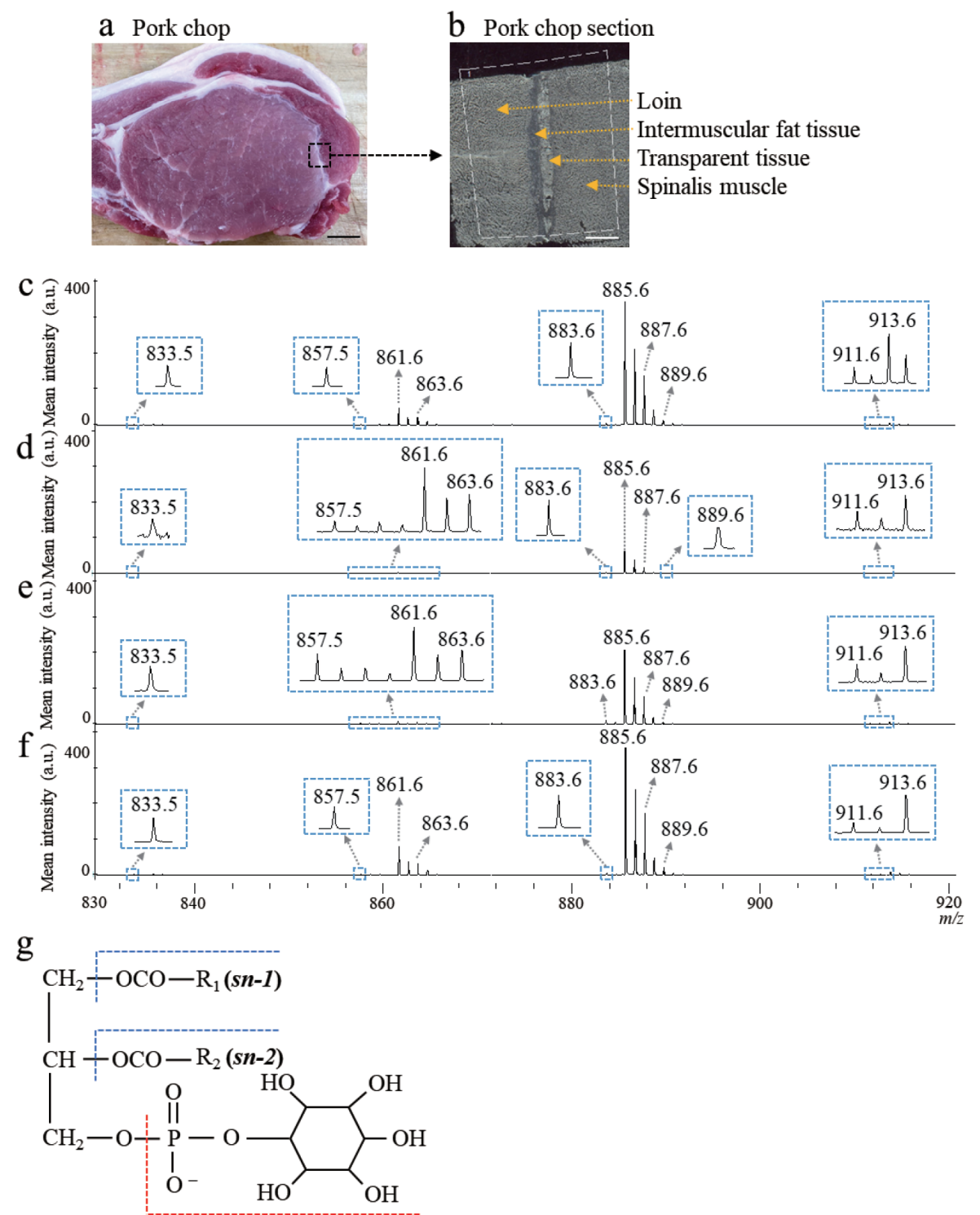

Fig. 1 Pork chop analysis using MALDI-MSI. (a) Optical image of the pork chop used in this study. The dotted square line marks the analyzed region. Scale bar $=10 \mathrm{~mm}$. (b) Optical image of pork section before MALDI-MSI analysis. Loin, Longissimus thoracis et lumborum muscle. Scale bar $=2 \mathrm{~mm}$. The mass spectra at $\mathrm{m} / z$ 830-920 of(c) loin, (d) intermuscular fat tissue, (e)transparent tissue, and(f) spinalis muscle. Peaks with $\mathrm{m} / z$ values indicate identified diacyl-phosphatidylinositol(PI) molecular species. (g) Structure of diacyl-PI molecular species. The dotted red line indicates the fragmentation position of the inositol phosphate $[\mathrm{M}-\mathrm{H}]^{-}$ion generated, whereas the dotted blue line indicates the fragmentation positions of two fatty acid $[\mathrm{M}-\mathrm{H}]^{-}$ions generated during tandem mass spectrometry. a. u., arbitrary units.

tetraenoic (22:4) acids, respectively, were detected (Table 1). Thus, $m / z 833.519,863.559,883.529,885.550,887.563$, 889.578, 911.577, and 913.577 were assigned as PI(diacyl 16:0/18:2) and PI (diacyl 18:0/16:2), PI (diacyl 18:0/18:1), PI (diacyl 18:0/20:5) and PI (diacyl 18:1/20:4), PI (diacyl 18:0/20:4), PI(diacyl 18:0/20:3), PI(diacyl 18:0/20:2), PI (diacyl 18:0/22:5), and PI(diacyl 18:0/22:4) $[\mathrm{M}-\mathrm{H}]^{-}$ions, respectively (Table 1). However, four PI molecular species had different retention times $(\mathrm{RT}): 7.5$, and $7.9 \mathrm{~min}$ for PI (diacyl 18:1/20:4); 12.1, 12.5, and 13.3 min for PI(diacyl 18:0/20:3); 14.2, and 15.1 min for PI(diacyl 18:0/20:2); and 9.4, 10.24, and $11.41 \mathrm{~min}$ for PI (diacyl 18:0/22:5) (Table 1). This result suggested the presence of structural isomers of PI(diacyl 18:1/20:4), PI (diacyl 18:0/20:3), PI (diacyl 18:0/20:2), and PI(diacyl 18:0/22:5) in the pork chop. The differences in RT might be due to the switching of fatty acid position at $s n-1$ and $s n-2$, or the positional change of the carbon-carbon double bond in the unsaturated fatty 
Table 1 Phosphatidylinositol(PI) molecular species in pork chop identified using LC-ESI-MS/MS.

\begin{tabular}{ccccc}
\hline $\begin{array}{c}\text { Precursor ion } m / z, \\
{[\mathrm{M}-\mathrm{H}]^{-}}\end{array}$ & Molecular species & $\begin{array}{c}\text { Product ion for assignment, } \\
\mathrm{m} / \mathrm{z}\end{array}$ & $\begin{array}{c}\text { Retention time, } \\
\text { min }\end{array}$ & $\begin{array}{c}\text { Relative intensity, } \\
\%\end{array}$ \\
\hline 833.519 & PI (diacyl 16:0/18:2) & $241.013,255.232,279.233$ & 8.5 & $0.21 \pm 0.01$ \\
& PI (diacyl 18:0/16:2) & $241.013,251.203,283.266$ & 8.9 & $0.17 \pm 0.01$ \\
& & & 7.7 & $0.36 \pm 0.01$ \\
\hline 857.517 & PI (diacyl 16:0/20:4) & $241.013,255.232,303.233$ & 11.6 & $8.27 \pm 0.23$ \\
\hline 861.553 & PI (diacyl 18:0/18:2) & $241.013,279.233,283.266$ & 14.5 & $3.64 \pm 0.11$ \\
\hline 863.559 & PI (diacyl 18:0/18:1) & $241.013,281.249,283.260$ & 14.5 \\
\hline 883.529 & PI (diacyl 18:0/20:5), & $241.013,283.266,301.220$ & 8.4 & $0.19 \pm 0.01$ \\
& PI (diacyl 18:1/20:4) & $241.013,281.249,303.233$ & 7.5 & $0.58 \pm 0.02$ \\
& & & 7.9 & $0.18 \pm 0.00$ \\
& & & & Total 0.95 \\
\hline 885.550 & PI (diacyl 18:0/20:4) & $241.013,283.266,303.233$ & 10.7 & $70.22 \pm 5.44$ \\
\hline 887.563 & PI (diacyl 18:0/20:3) & $241.013,283.260,305.248$ & 12.1 & $5.42 \pm 0.18$ \\
& & & 12.5 & $1.25 \pm 0.04$ \\
& & & 13.3 & $7.35 \pm 0.28$ \\
& & & & Total 14.02 \\
\hline 889.578 & PI (diacyl 18:0/20:2) & $241.013,283.260,307.263$ & 14.2 & $0.42 \pm 0.01$ \\
& & & 15.1 & $0.21 \pm 0.00$ \\
& & & & Total 0.63 \\
\hline 911.577 & PI (diacyl 18:0/22:5) & $241.013,283.260,329.250$ & 9.4 & $0.03 \pm 0.01$ \\
& & & 10.2 & $0.21 \pm 0.01$ \\
& & & 11.4 & $0.08 \pm 0.00$ \\
& & & 12.7 & $1.31 \pm 0.06$ \\
\hline 913.577 & PI (diacyl 18:0/22:4) & $241.013,283.260,331.266$ & & \\
\hline
\end{tabular}

PI molecular species were identified using LC-ESI-MS/MS analysis.

Relative intensity was calculated by each peak area using LC-ESI-MS analysis. The data are represented as the mean \pm standard deviation $(\mathrm{n}=3)$.

Table 2 Phosphatidylinositol(PI) molecular species identified by MALDI-MS/MS analysis.

\begin{tabular}{|c|c|c|c|}
\hline $\begin{array}{l}\text { Precursor ion } m / z \text {, } \\
\qquad[\mathrm{M}-\mathrm{H}]^{-}\end{array}$ & Molecular species & $\begin{array}{l}\text { Product ion for } \\
\text { assignment, } m / z\end{array}$ & $\begin{array}{c}\text { Relative intensity, } \\
(\%)\end{array}$ \\
\hline 833.5 & $\begin{array}{l}\text { PI (diacyl 16:0/18:2) } \\
\text { PI (diacyl 18:0/16:2) }\end{array}$ & $241,251,255,279,283$ & $0.23 \pm 0.01$ \\
\hline 857.5 & PI (diacyl 16:0/20:4) & $241,255,303$ & $0.48 \pm 0.03$ \\
\hline 861.6 & PI (diacyl 18:0/18:2) & $241,279,281,283$ & $7.27 \pm 0.27$ \\
\hline 863.6 & PI (diacyl 18:0/18:1) & $241,281,283$ & $3.95 \pm 0.29$ \\
\hline 883.6 & $\begin{array}{l}\text { PI (diacyl 18:0/20:5), } \\
\text { PI (diacyl 18:1/20:4) }\end{array}$ & $241,281,283,301,303$ & $1.46 \pm 0.16$ \\
\hline 885.6 & PI (diacyl 18:0/20:4) & $241,283,303$ & $61.93 \pm 3.96$ \\
\hline 887.6 & PI (diacyl 18:0/20:3) & $241,283,305$ & $20.93 \pm 1.37$ \\
\hline 889.6 & PI (diacyl 18:0/20:2) & $241,283,307$ & $2.41 \pm 0.04$ \\
\hline 911.6 & PI (diacyl 18:0/22:5) & $241,283,329$ & $0.34 \pm 0.02$ \\
\hline 913.6 & PI (diacyl 18:0/22:4) & $241,283,331$ & $1.22 \pm 0.05$ \\
\hline
\end{tabular}

MALDI-MS/MS analysis was performed directly on pork chop sections. The relative intensities of individual PI molecular species are presented as the mean \pm standard error $(n=3)$. 


\section{H. Enomoto, S. Takeda and H. Hatta}

acids at $s n-1$ and $s n-2$. Overall, the most predominant diacyl-PI molecular species was PI (diacyl 18:0/20:4), followed by PI(diacyl 18:0/18:3), PI(diacyl 18:0/18:2), and PI(diacyl 18:0/18:1), and their ratios of relative intensities were $70.22,14.02,8.27$, and $3.64 \%$, respectively. The top four PI molecular species accounted for $96.15 \%$ of total PI molecular species. This result was consistent with a previous study on LC-ESI-MS/MS analysis of phospholipids in pork ${ }^{1)}$.

Further, we performed MALDI-MS/MS analysis of the pork sections and confirmed the fragment ion peaks corresponding to the $m / z$ values of inositol phosphate and fatty acid $[\mathrm{M}-\mathrm{H}]^{-}$ions detected by LC-ESI-MS/MS analysis (Table 2). The most predominant diacyl-PI molecular species was PI(diacyl 18:0/20:4), followed by PI(diacyl 18:0/18:3), PI(diacyl 18:0/18:2), and PI(diacyl 18:0/18:1), and their ratios of relative intensities were $61.93,20.93$, 7.27 , and $3.95 \%$, respectively. These top four PI molecular species accounted for $94.08 \%$ of total PI molecular species. These results were similar to that of LC-ESI-MS analysis, suggesting that MALDI-MSI can analyze the relative abundance of PI molecular species in pork chop with the equivalent accuracy as LC-ESI-MS analysis.

\subsection{Distribution and composition of diacyl-PI molecular species in pork sections}

To investigate the distribution and composition of diacylPI molecular species in loin, intermuscular fat tissue, transparent tissue, and spinalis muscle of the pork chop sections (Fig. 1b), we reconstructed ion images using their $m / z$ values. PI molecular species containing 20:3, 20:4, $22: 4$, and $22: 5$ at the $s n-2$ position; namely, PI (diacyl 16:0/20:4), PI (diacyl 18:0/20:3), PI(diacyl 18:0/20:4), PI (diacyl 18:0/22:4), PI(diacyl 18:0/20:5), and PI(diacyl 18:1/20:4) and PI (diacyl 18:0/20:5), respectively, were mainly abundant in the loin, transparent tissue, and spinals muscle (Figs. 2b, 2f-2j), whereas those containing 18:1, $18: 2$, and $20: 2$ at the $s n-2$ position; namely, PI (diacyl 16:0/18:2) and PI (diacyl 18:0/16:2), PI (diacyl 18:0/18:1), PI (18:0/18:2), and PI(diacyl 18:0/20:2), respectively, were mainly abundant in the two muscle tissues(Figs. 2a, 2c-2e). Interestingly, the concentration of each PI molecular species differed among the four tissues. Therefore, the mean detection intensities of each PI molecular species in the four tissues were calculated to compare their concentrations among these tissues. The concentrations of PI (diacyl 16:0/18:2) and PI (diacyl 18:0/16:2), PI (diacyl 18:0/20:4), and PI(diacyl 18:0/20:3) were the highest in both loin and spinalis muscle (Fig. 3a). The concentrations of PI (diacyl 16:0/20:4), and PI (diacyl 18:0/20:5) and PI (diacyl 18:1/20:4)were the highest in transparent tissue, whereas PI(diacyl 18:0/22:5) was the highest concentration in transparent tissue and spinalis muscle. The concentrations of PI(diacyl 18:0/18:2), PI (diacyl 18:0/18:1), PI (diacyl 18:0/20:2), and PI(diacyl 18:0/22:4) were the

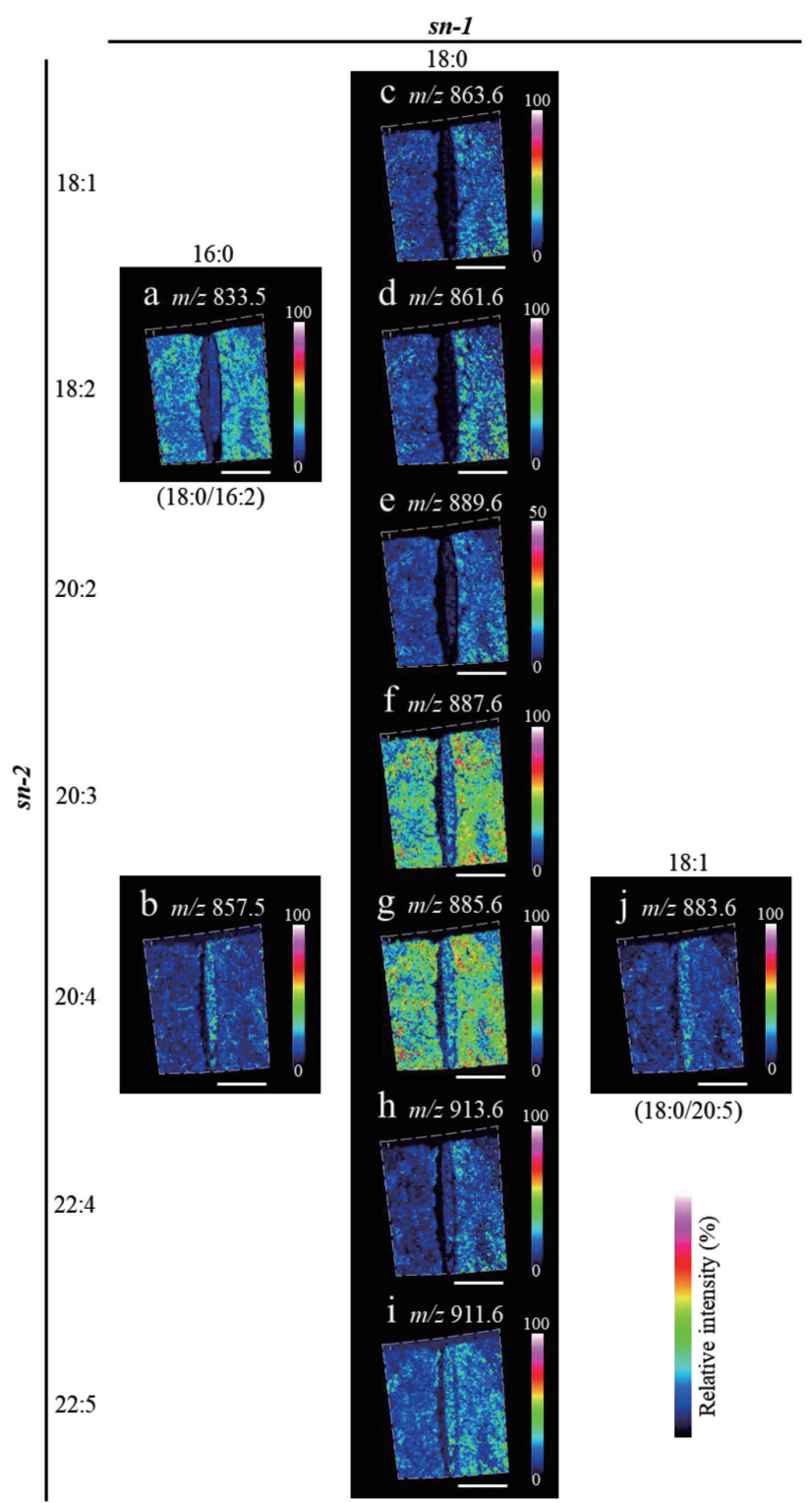

Fig. 2 Visualization of identified diacyl-phosphatidylinositol molecular species in pork chop sections. ( $\mathrm{a}-\mathrm{j})$ Representative ion images arranged according to their fatty acid compositions. Fatty acid compositions at the $s n-1$ position are arranged along the $\mathrm{x}$-axis, and those at the $s n-2$ position are arranged along the $y$-axis. The color gradient of the ion images shows the relative intensities of signals detected for each section. The dotted white line shows the analyzed region. Scale bar $=2 \mathrm{~mm}$.

highest in spinalis muscle. These results suggested that the distribution patterns of diacyl-PI molecular species in pork chop tissues were dependent on fatty acid compositions at the $s n-2$ position.

To compare the total content of diacyl-PI molecular species in loin, intramuscular fat tissue, transparent tissue, 

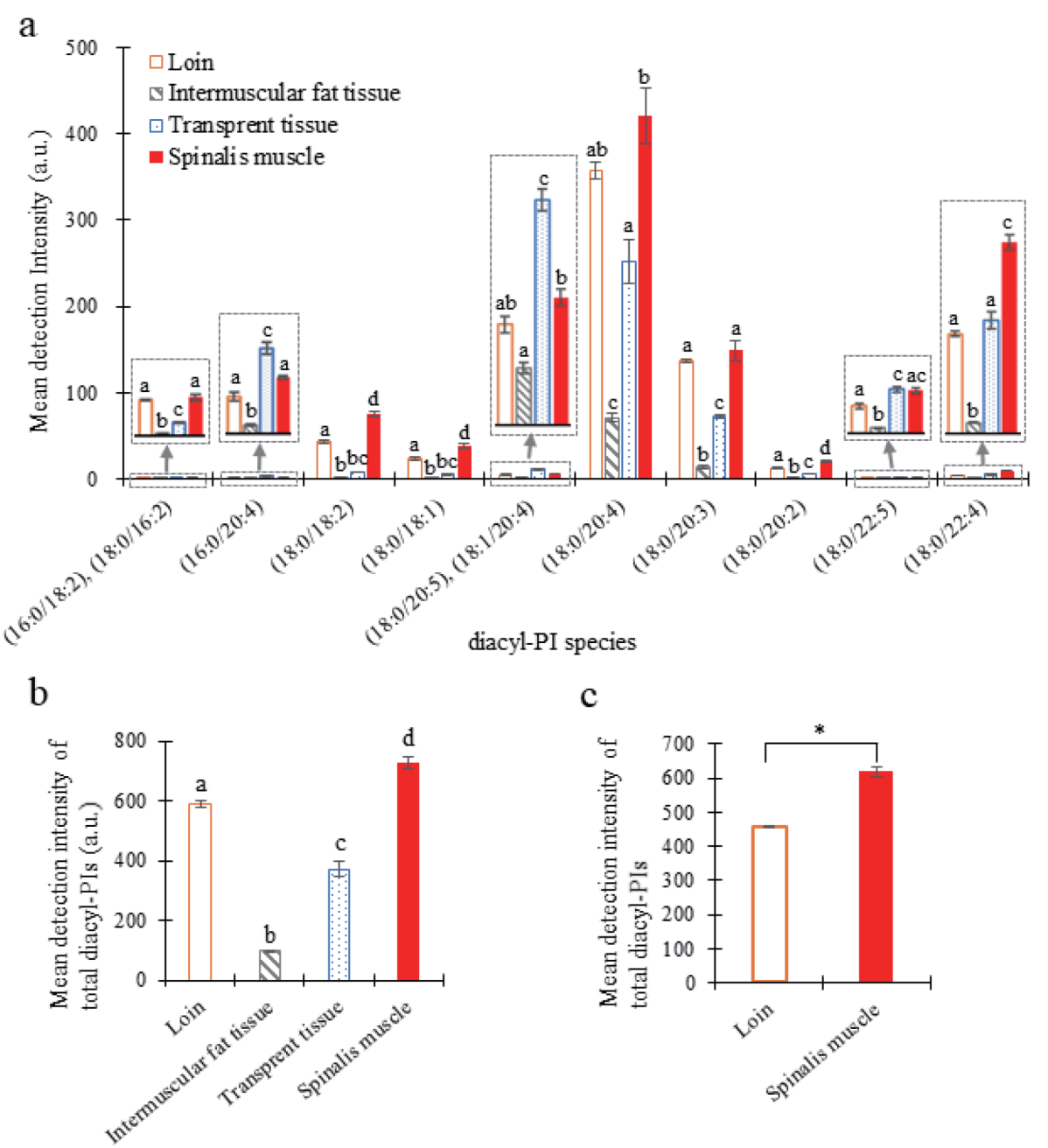

Fig. 3 Concentrations of identified diacyl-phosphatidylinositol(PI) molecular species in Longissimus thoracis et lumborum muscle (loin), intermuscular fat tissue, transparent tissue, and spinalis muscle of pork chop sections. (a) Mean detection intensities of each diacyl-PI molecular species in the four tissues using MALDI-MSI. (b) Total mean detection intensities of diacyl-PI molecular species in the four tissues using MALDI-MSI. The data are expressed as the mean \pm standard error $(n=3)$. Mean values with different letters indicate significant differences $(p<0.05$; Tukey' s post hoc test). a. u., arbitrary units. (c) Total mean areas of diacyl-PI molecular species in the loin and spinalis muscle using LC-ESI-MS. The data are expressed as the mean \pm standard deviation $(\mathrm{n}=3)$. * $p<0.05$; Student's $t$-test.

and spinalis muscle, their mean detection intensities obtained by MALDI-MSI analysis were combined. The total content of diacyl-PI molecular species of both muscle tissues was significantly higher than those of other two tissues (Fig. 3b). In addition, the content of diacyl-PI molecular species in spinalis muscle was 1.24 times significantly higher than that in loin. To verify the result of MALDI-MSI analysis, the mean detection intensities of diacyl-PI molecular species between loin and spinalis muscle were compared using LC-ESI-MS analysis. The content of diacylPI molecular species in spinalis muscle was 1.35 times significantly higher than that in loin (Fig. 3c). This result was similar to that of MALDI-MSI analysis, suggesting that the accuracy of MALDI-MSI analysis for both muscle tissues was equivalent to that of LC-ESI-MS analysis.

In the present study, we identified 12 PI molecular species in four different pork chop tissues using LC-ESIMS/MS and MALDI-MS/MS analysis. In addition, we revealed characteristic distribution patterns of PI molecular species in the four tissues of pork chop.

There are nine possible stereoisomers of inositol, and PI molecular species generally have myo-inositols ${ }^{18)}$. It has been suggested that myo-inositol deficiency causes cranial neural tube defects (NTDs), such as anencephaly and spina bifida in several rodent models ${ }^{18)}$. Myo-inositol has been studied in humans for a range of conditions and appears to 


\section{H. Enomoto, S. Takeda and H. Hatta}

be safe for use in human pregnancy. In cohort studies from Italy and the United Kingdom, supplementation of myoinositol with folic acid to woman showed preventive effects against NTDs ${ }^{18)}$. Previous studies have reported the role of myo-inositol in the management of polycystic ovary syndrome (PCOS) in women as well as in the prevention and treatment of gestational diabetes mellitus (GDM) ${ }^{19,20)}$. The anticancer effect of myo-inositol has also been demonstrated in various experimental models ${ }^{21)}$. In the present study, we found that PI molecular species in pork chop tissues were most abundant in spinalis muscle, followed by loin, transparent tissue, and intermuscular fat tissue (Figs. $3 b, 3 c)$, respectively. Further analysis using animal and cell culture models are needed to assess the protective effects of each tissue on diseases such as NTDs, PCOS, GDM, and cancer.

Other studies have reported various bioactive molecules associated with both health-promoting effects and unhealthiness in pork ${ }^{15-17,24)}$. Pork is commonly consumed in processed forms such as steak, ham, and sausage. Therefore, to assess the health-related properties of each pork tissue, spatial analysis of PI molecular species and other bioactive molecules in various types of raw and processed pork is necessary.

\section{Conclusion}

We demonstrated that the combination of LC-ESI-MS and MALDI-MSI analysis is useful for the spatial analysis of PI molecular species in pork chop tissues. The analytical approach used in this study as well as the spatial information of PI molecular species provided here would contribute to our knowledge on the beneficial properties, including health benefits, of pork and other meats.

\section{Acknowledgments}

We thank Ms. Thủy Nguyễn, and Mr. Richard Tjeng for their assistance with MALDI-MSI analysis. This work was supported by Advanced Comprehensive Research Grants of Teikyo University (H.E.). We thank Editage (www. editage.jp) for English language editing.

\section{Conflict of Interest}

The authors declare no conflicts of interest.

\section{References}

1) Boselli, E.; Pacetti, D.; Curzi, F.; Frega, N.G. Determi- nation of phospholipid molecular species in pork meat by high performance liquid chromatography-tandem mass spectrometry and evaporative light scattering detection. Meat Sci. 78, 305-313 (2008).

2) Norris, C.; Fong, B.; MacGibbon, A.; McJarrow, P. Analysis of phospholipids in rat brain using liquid chromatography-mass spectrometry. Lipids 44, 1047-1054 (2009).

3) Caprioli, R.M.; Farmer, T.B.; Gile, J. Molecular imaging of biological samples: Localization of peptides and proteins using MALDI-TOF MS. Anal. Chem. 69, 47514760 (1997).

4) Cornett, D.S.; Reyzer, M.L.; Chaurand, P.; Caprioli, R.M. MALDI imaging mass spectrometry: Molecular snapshots of biochemical systems. Nat. Methods 4, 828833 (2007).

5) Sugiura, Y.; Shimma, S.; Setou, M. Two-step matrix application technique to improve ionization efficiency for matrix-assisted laser desorption/ionization in imaging mass spectrometry. Anal. Chem. 78, 8227-8235 (2006).

6) Rešetar Maslov, D.; Svirkova, A.; Allmaier, G.; Marchetti-Deschamann, M.; Kraljević Pavelić, S. Optimization of MALDI-TOF mass spectrometry imaging for the visualization and comparison of peptide distributions in dry-cured ham muscle fibers. Food Chem. 283, 275286 (2019).

7) Enomoto, H.; Sato, K.; Miyamoto, K.; Ohtsuka, A.; Yamane, H. Distribution analysis of anthocyanins, sugars, and organic acids in strawberry fruits using matrix-assisted laser desorption/ionization-imaging mass spectrometry. J. Agric. Food Chem. 66, 4958-4965(2018).

8) Enomoto, H.; Nirasawa, T. Localization of flavan-3-ol species in peanut testa by mass spectrometry imaging. Molecules 25, 2373(2020).

9) Yukihiro, Y.; Zaima, N. Application of mass spectrometry imaging for visualizing food components. Foods 9, 575 (2020).

10) Goto-Inoue, N.; Hayasaka, T.; Zaima, N.; Setou M. Imaging mass spectrometry for lipidomics. Biochim. Biophys. Acta 1811, 961-969(2011).

11) Zaima, N.; Goto-Inoue, N.; Hayasaka, T.; Enomoto, H.; Setou, M. Authenticity assessment of beef origin by principal component analysis of matrix-assisted laser desorption/ionization mass spectrometric data. Anal. Bioanal. Chem. 400, 1865-1871 (2011).

12) Enomoto, H.; Takeda, S.; Hatta, H.; Zaima, N. Tissuespecific distribution of sphingomyelin species in pork chop revealed by matrix-assisted laser desorption/ionization-imaging mass spectrometry. J. Food Sci. 84, 1758-1763 (2019).

13) Enomoto, H.; Furukawa, T.; Takeda, S.; Hatta, H.; Zaima, N. Unique distribution of diacyl-, alkylacyl-, and alkenylacyl-phosphatidylcholine species visualized in 
pork chop tissues by matrix-assisted laser desorption/ ionization-mass spectrometry imaging. Foods 9, 205 (2020).

14) Kawashima, M.; Tokiwa, M.; Nishimura, T.; Kawata, Y.; Sugimoto, M.; Kataoka, T.R.; Sakurai, T.; Iwaisako, K.; Suzuki, E.; Hagiwara, M.; Harris, A.L.; Toi, M. Highresolution imaging mass spectrometry combined with transcriptomic analysis identified a link between fatty acid composition of phosphatidylinositols and the immune checkpoint pathway at the primary tumour site of breast cancer. Br. J. Cancer. 122, 245-257 (2020).

15) De Smet, S.; Vossen, E. Meat: The balance between nutrition and health. A review. Meat Sci. 120, 145-156 (2016).

16) Listrat, A.; Lebret, B.; Louveau, I.; Astruc, T.; Bonnet, M.; Lefaucheur, L.; Picard, B.; Bugeon, J. How muscle structure and composition influence meat and flesh quality. Sci. World J. 2016, 3182746 (2016).

17) Ma, X.; Jiang, Z.; Lai, C. Significance of increasing n-3 PUFA content in pork on human health. Crit. Rev. Food Sci. Nutr. 56, 858-870(2016).

18) Greene, N.D.; Leung, K.Y.; Copp, A.J. Inositol, neural tube closure and the prevention of neural tube defects. Birth Defects Res. 109, 68-80 (2017).

19) D’Anna, R.; Santamaria, A.; Alibrandi, A.; Corrado, F.; DI Benedetto, A.; Facchinetti, F. Myo-inositol for the prevention of gestational diabetes mellitus. A brief review. J. Nutr. Sci. Vitaminol. 65, S59-S61 (2019).

20) Gateva, A.; Unfer, V.; Kamenov, Z. The use of inositol $(\mathrm{s})$ isomers in the management of polycystic ovary syndrome: A comprehensive review. Gynecol. Endocrinol. 34, 545-550 (2018).

21) Vucenik, I. Anticancer properties of inositol hexaphosphate and inositol: An overview. J. Nutr. Sci. Vitaminol. 65, S18-S22(2019).

22) Bligh, E.G., Dyer, W.J. A rapid method of total lipid extraction and purification. Can. J. Biochem. Physiol. 37, 911-917 (1959).

23) Dean, J.M.; Lodhi, I.J. Structural and functional roles of ether lipids. Protein Cell 9, 196-206 (2018).

24) Takeda, S.; Kaneko, S.; Sogawa, K.; Ahhmed, A.M.; Enomoto, H.; Kawarai, S.; Taira, K.; Mizunoya, W.; Minami, M.; Sakata, R. Isolation, evaluation, and identification of angiotensin I-converting enzyme inhibitory peptides from game meat. Foods 9, 1168(2020).

CC BY 4.0 (Attribution 4.0 International). This license allows users to share and adapt an article, even commercially, as long as appropriate credit is given. That is, this license lets others copy, distribute, remix, and build upon the Article, even commercially, provided the original source and Authors are credited. 